\title{
Strongyloides stercoralis - a Legacy from the Past
}

\author{
$\underline{\text { Inês Chora }}{ }^{1}$, Rodrigo Pimentel ${ }^{2}$, Paula Dias ${ }^{1}$, Fernando Friões ${ }^{1}$, Sérgio Silva $^{1}$ \\ ${ }^{1}$ Internal Medicine Department, Centro Hospitalar de São João, Porto, Portugal \\ ${ }^{2}$ Intensive Medicine Department, Centro Hospitalar de São João, Porto, Portugal
}

Received: 15/09/2015

Accepted: 30/09/2015

Published: 16/10/2015

How to cite this article: Chora I, Pimentel R, Dias P, Fernando Friões F, Silva S. Strongyloides stercoralis - a legacy from the past.

EJCRIM 2015;2:doi:10.12890/2015_000286

Conflicts of Interests: The authors declare that there are no competing interests.

Aknowledgements: The authors would like to acknowledge the staff in the Intensive Care Unit of Infectious Diseases, in the Department of Clinical Pathology, the Microbiology Division and the Department of Anatomical Pathology at Centro Hospitalar de São João, Porto, Portugal.

This article is licensed under a Commons Attribution Non-Commercial 4.0 License

\section{ABSTRACT}

The authors present the case of a 65-year-old Caucasian man who had previously lived in Guinea-Bissau. The patient was diagnosed in Portugal with an anti-neutrophil cytoplasmic antibody-associated vasculitis and started on corticosteroids plus cyclophosphamide, with clinical improvement. Some months later, his general status deteriorated, iatrogenic bicytopenia developed and immunosuppressive drugs were tapered. Microbiological tests identified numerous larvae and eggs of Strongyloides stercoralis in various biological samples, and a diagnosis of hyperinfection syndrome was established. The patient was started on antihelminthic drugs but developed septic shock and died. This case highlights a rare and severe complication of immunosuppression in developed countries.

\section{LEARNING POINTS}

- Strongyloides stercoralis has the unique ability to replicate in the human host and so can persist for decades after exposure in endemic areas.

- Strongyloidiasis must be considered in patients with epidemiological risk factors, regardless of the time since exposure.

- Patients at risk for strongyloidiasis should be treated empirically with oral ivermectin or albendazol, even if they are asymptomatic and/ or without blood eosinophilia, before starting immunosuppressive agents.

\section{KEYWORDS}

Anti-neutrophil cytoplasmic antibody-associated vasculitis; immunosuppression, Strongyloides stercoralis.

\section{CASE PRESENTATION}

The authors report the case of a 65-year-old Caucasian man with dyslipidaemia and hyperuricaemia. He had lived in Guinea-Bissau from 1970 to 1973 during military service but had no history of recent travel.

Four months before his last admission, a peripheral sensory-motor polyneuropathy was diagnosed and interpreted as secondary to lumbosacral nerve compression on a vertebral computed tomography (CT) scan. Gabapentin and amitriptyline were started and the patient was referred to neurosurgery for consultation.

One month later, the patient was admitted to a stroke unit with a posterior circulation ischaemic stroke, with no evidence of stroke on cerebral CT scan; studies revealed normocytic normochromic (NN) anaemia, increased plasma creatinine (1.7 mg/dl), an increased 
erythrocyte sedimentation rate (ESR; $63 \mathrm{~mm} / \mathrm{s}$ ), positive antinuclear antibodies (ANA; 1/320) and increased rheumatoid factor (1080 IU/ $\mathrm{ml})$. He was discharged under aspirin and statin and referred for cerebrovascular consultation.

The patient was readmitted 2 weeks later to an internal medicine ward for acute and rapidly progressive renal failure (non-oliguric and non-obstructive), asthenia, anorexia, weight loss, dizziness and gait ataxia. He had a purpuric rash on his lower limbs, NN anaemia, a normal leucogram and plasma creatinine $2.3 \mathrm{mg} / \mathrm{dl}$. Systemic vasculitis with renal, cutaneous, cerebral and neuronal involvement was suspected and confirmed by the finding of anti-neutrophil cytoplasmic antibodies-proteinase 3 (ANCA-PR3; $150 \mathrm{U} / \mathrm{ml}$ ). A sural nerve biopsy showed vasculitic activity (Figs. 1 and 2).

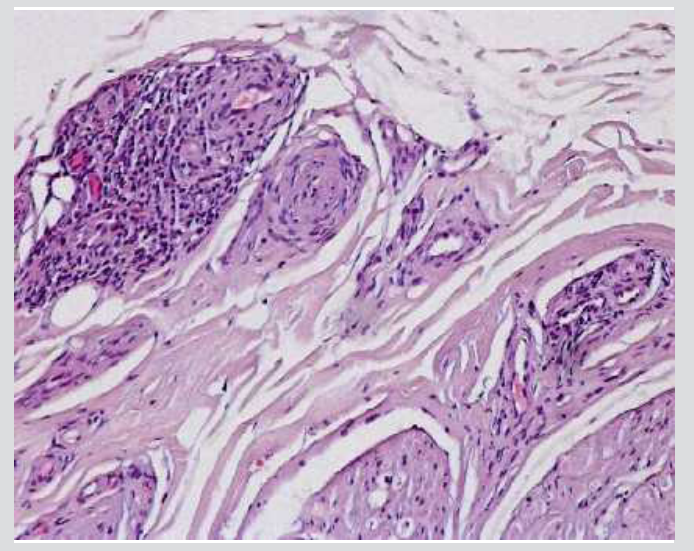

Figure 1: Nerve, transversal cut (eosin hematoxylin). Vessels surrounded by chronic inflammatory infiltrates, sometimes invading vascular wall - chronic axonal neuropathy, in activity, of vasculitic aetiology (courtesy of Dr. Ligia Castro, Department of Anatomical Pathology, Centro Hospitalar de São João).

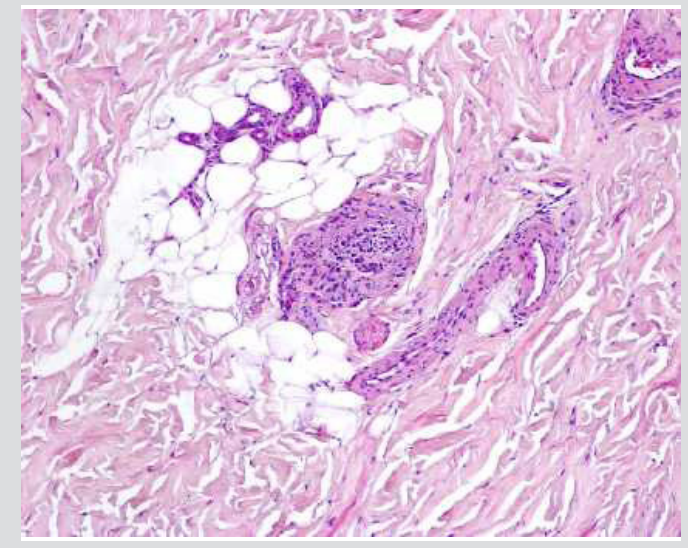

Figure 2: Skin (eosin hematoxylin). Vasculitis of subcutaneous cellular tissue is shown (courtesy of Dr. Ligia Castro, Department of Anatomical Pathology, Centro Hospitalar de São João).

The patient had a Birmingham Vasculitis Activity Score of 25 and a Revised Five-Factor Score of 2. He was started on oral prednisone 1 mg/ $\mathrm{kg} /$ day and cyclophosphamide $1.5 \mathrm{mg} / \mathrm{kg}$ /day (plus omeprazole, calcium, vitamin D and prophylactic cotrimoxazol) and discharged 8 days later with improved renal function and without skin lesions. He was prescribed physiotherapy for gait rehabilitation.

On follow-up consultation, the patient's general status and renal function had improved, ANCA was decreased and ESR was normal. Thoracic and nasal CT scans were normal. Prednisone tapering was started. One month later, the patient was readmitted for asthenia, dysphagia, severe obstipation with gastric intolerance and bicytopenia. Plasma creatinine was normal and ANCA was negative. Abdominal radiography showed colic distension without hydroaeric levels. Cyclophosphamide was stopped and a faster tapering of prednisone was started. The patient showed progressive clinical deterioration and, on day 4, was transferred to an intermediate care unit because of bilateral pneumonia with hypoxaemic respiratory failure. Extensive microbiological samples were obtained and large-spectrum antibiotics were prescribed. Because of gastric intolerance, an upper endoscopy for nasojejunal tube placement was performed, with duodenal biopsies. Numerous larvae and eggs of Strongyloides stercoralis were identified on bronchoalveolar fluid, gastric juice, stool and duodenal samples (Figs. 3 and 4). A diagnosis of S. stercoralis hyperinfection syndrome (SHS) was established and the patient was started on oral albendazol and parenteral ivermectin. However, he died on day 17 in the intensive care unit from septic shock and multiorgan failure.

\section{DISCUSSION}

We describe this case in order to highlight a very unusual complication of immunosuppression in a developed country and to illustrate the importance of multidisciplinary management in complex clinical scenarios.

The presence of systemic vasculitis should be considered in patients with unexplained systemic symptoms and single or multiorgan dysfunction. While early deaths in vasculitis are due to disease activity, late deaths are frequently due to complications of immunosuppressive therapy ${ }^{[1]}$.

S. stercoralis is an intestinal helminth which infects humans through skin contact with contaminated soil ${ }^{[2,3]}$. It is endemic in the tropics and subtropics, such as Guinea-Bissau, and can survive for decades in a single host because it can complete its life cycle inside the human body (autoinfection), without passing out into the environment ${ }^{[2,4]}$. 


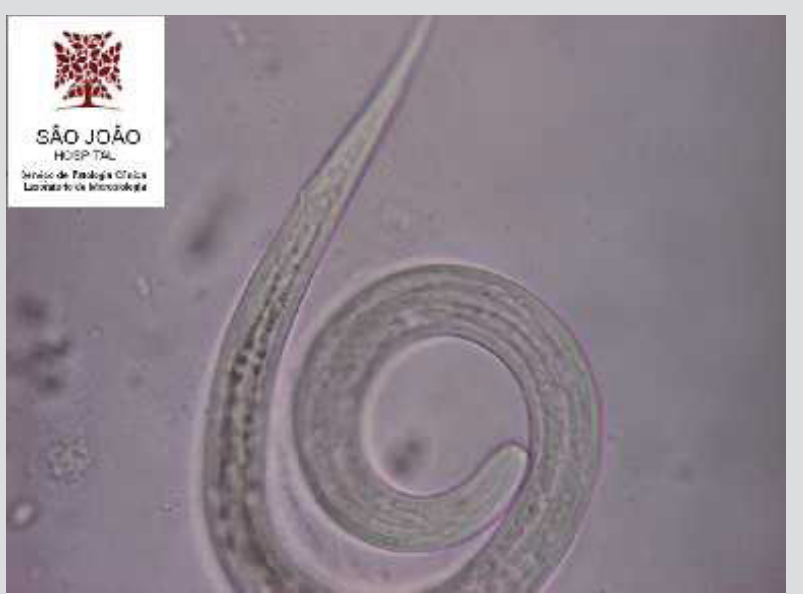

Figure 3: A filariform larva of Strongyloides stercoralis isolated from the patient's gastric juice is shown (courtesy of Dr. Manuela Ribeiro, Department of Clinical Pathology Division of Microbiology, Centro Hospitalar de São João).

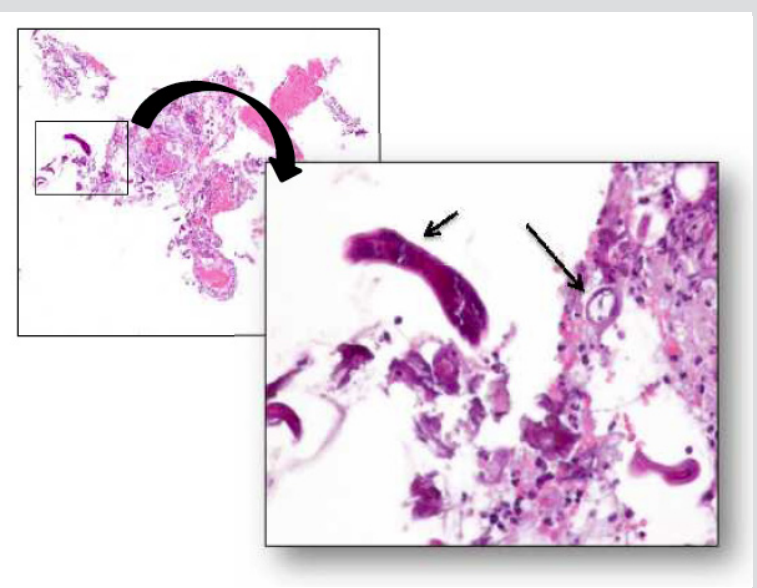

Figure 4: Duodenal mucosa (haematoxylin and eosin). A Strongyloides stercoralis larva and egg (left and right small arrows, respectively) are shown (courtesy of Dr. Francisco Beça, Department of Anatomical Pathology, Centro Hospitalar de São João).

Accordingly, risk for strongyloidiasis must be considered in anyone who has been in endemic areas, regardless of the time since exposure (this patient had lived in Guinea-Bissau in the early 1970s).

Strongyloidiasis may present with cutaneous or gastrointestinal symptoms but is asymptomatic in over $60 \%$ of cases and only suggested by peripheral blood eosinophilia ${ }^{[2]}$. Immunosuppressed patients with chronic strongyloidiasis are at high risk of developing SHS, a lifethreatening complication where overproliferation of larvae causes sepsis and multiorgan failure ${ }^{[2-4]}$, as occurred in this case. Therefore, at-risk patients should be identified before immunosuppression is started. Glucocorticoids are a major risk factor for SHS, even when given in short courses. Eosinophilia is usually absent during hyperinfection ${ }^{[4]}$.

If strongyloidiasis is diagnosed early, it is easily treatable with oral ivermectin (cure rate of 94-100\%) ${ }^{[2,5]}$. However, patients with chronic strongyloidiasis usually have a low parasite load and irregular larval output, making the condition very difficult to diagnose ${ }^{[5]}$. Patients with previous contact with endemic areas and either compatible symptoms or blood eosinophilia should be investigated ${ }^{[2]}$. In our patient, despite the epidemiological context, there were no suggestive clinical features or documented eosinophilia, even before corticosteroids were started.

SHS is difficult to treat and has a high mortality rate $(75-80 \%)^{[5]}$. Management consists of antihelminthic treatment, antibiotics for Gramnegative bacterial sepsis, multiorgan support and a reduction in immunosuppressive treatment when possible. Oral ivermectin is the drug of choice. Parenteral ivermectin has been shown to be as effective and should be used when the oral route is unavailable (although, unfortunately, in our country, it is only available as a veterinary preparation) ${ }^{[2]}$. Oral albendazol is another option. In this patient, parenteral ivermectin and oral albendazol were administered, as well as large-spectrum antibiotics and multiorgan support provided, but without success.

In light of the high mortality of immunosuppressed patients with SHS, empirical treatment with ivermectin or albendazol (when ivermectin is not available) should be offered before immunosuppression is started, to prevent long-term complications in patients at risk for strongyloidiasis, even in the absence of symptoms and/or eosinophilia.

\section{REFERENCES}

1. Villa-Forte A, European League Against Rheumatism/European Vasculitis Study Group. European League Against Rheumatism/European Vasculitis Study Group recommendations for the management of vasculitis. Curr Opin Rheumatol 2010;22:49-53.

2. Greaves D, Coggle S, Pollard C, Aliyu SH, Moore EM. Strongyloides stercoralis infection. BMJ 2013;347:f4610.

3. Kassalik M, Mönkemüller K. Strongyloides stercoralis hyperinfection syndrome and disseminated disease. Gastroenterol Hepatol 2011;7:766-768.

4. Pukkila-Worley R, Nardi V, Branda JA. Case records of the Massachusetts General Hospital. Case 28-2014. A 39-year-old man with a rash, headache, fever, nausea, and photophobia. N Engl J Med 2014;371:1051-1060.

5. Montes M, Sawhney C, Barros N. Strongyloides stercoralis: there but not seen. Curr Opin Infect Dis 2010;23:500-504. 